\title{
Fetal androgens and schizophrenia:
}

\section{Commentary on van Os and Kapur (Lancet 2009; 374: 635-45)}

Martin Voracek

This manuscript version: August 27, 2009

Martin Voracek, D.Sc., D.M.Sc., Ph.D., Department of Basic Psychological Research, School of Psychology, University of Vienna, Liebiggasse 5, Rm 03-46, A-1010 Vienna, Austria.

E-Mail: martin.voracek@univie.ac.at 


\section{Fetal androgens and schizophrenia:}

\section{Commentary on van Os and Kapur (Lancet 2009; 374: 635-45)}

The Seminar on schizophrenia by Jim van Os and Shitij Kapur ${ }^{1}$ is a rich source of current knowledge on the topic and highly useful for generalists. Under prenatal risk factors for schizophrenia, the authors list hypoxia and maternal infection, stress, and malnutrition. To this altered sex-hormone profiles might be added.

Sex differences in the lifetime prevalence, incidence, age of onset, and course of schizophrenia are well-known and have partly been attributed to possible sex-hormone influences. Indeed, several studies (reviewed in ref. 2) have shown lower circulating sex-hormone levels in schizophrenic patients (low testosterone levels in men and low estradiol levels in women).

A role of fetal androgens for schizophrenia has been suggested by recent research on the second-to-fourth digit ratio (2D:4D), a widely studied biomarker for prenatal testosterone. ${ }^{3}$ Human 2D:4D is sexually differentiated (lower in males than in females), and sex and individual differences in 2D:4D emerge prenatally and are preserved during the growth phases of postnatal life. ${ }^{3}$ Lower (male-typical) 2D:4D is associated with higher sensitivity to testosterone (i.e., functional polymorphisms in the androgen receptor gene) and a higher testosterone-to-estradiol ratio, as assayed from the amniotic fluid. ${ }^{3}$

Initial findings ${ }^{4}$ of a higher (female-typical) 2D:4D in both male and female schizophrenic patients, relative to same-sex normal healthy population controls, have been replicated by several subsequent reports (reviewed in ref. 5). All in all, the above lines of evidence point to a role of disturbed sex-hormone profiles, already arising in utero, in the pathways leading to the neurodevelopmental disorder schizophrenia. 


\section{References}

1 van Os J, Kapur S. Schizophrenia. Lancet 2009; 374: 635-45.

2 Voracek M. Who wants to believe? Associations between digit ratio (2D:4D) and paranormal and superstitious beliefs. Pers Individ Dif 2009; 47: 105-9.

3 McIntyre $\mathrm{MH}$. The use of digit ratios as markers for perinatal androgen action. Reprod Biol Endocrinol 2006; 4: 10.

4 Arató M, Frecska E, Beck C, et al. Digit length pattern in schizophrenia suggests disturbed prenatal hemispheric lateralization. Prog Neuropsychopharmac Biol Psychiatry 2004; 28: 1914.

5 Voracek M. Digit ratio (2D:4D) as a marker for mental disorders: low (masculinized) 2D:4D in autism-spectrum disorders, high (feminized) 2D:4D in schizophrenic-spectrum disorders. Behav Brain Sci 2008; 31: 283-4. 